\title{
Attributing Scientific and Technological Progress: The Case of Holography
}

\author{
Sean F. Johnston
}

Holography, the three-dimensional imaging technology, was portrayed widely as a paradigm of progress during its decade of explosive expansion 1964-73, and during its subsequent consolidation for commercial and artistic uses up to the mid 1980s. An unusually seductive and prolific subject, holography successively spawned scientific insights, putative applications and new constituencies of practitioners and consumers. Waves of forecasts, associated with different sponsors and user communities, cast holography as a field on the verge of success-but with the dimensions of success repeatedly refashioned. This retargeting of the subject represented a degree of cynical marketeering, but was underpinned by implicit confidence in philosophical positivism and faith in technological progressivism. Each of its communities defined success in terms of expansion, and anticipated continual progressive increase. This paper discusses the contrasting definitions of progress in holography, and how they were fashioned in changing contexts. Focusing equally on reputed 'failures' of some aspects of the subject, it explores the varied attributes by which success and failure were linked with progress by different technical communities. This important case illuminates the peculiar post-World War II environment that melded the military, commercial and popular engagement with scientific and technological subjects, and the competing criteria by which they assessed the products of science.

Keywords: Holography; Hologram; Progress; Expansion; Success; Failure; Forecasting; Dennis Gabor; Conductron; Kip Siegel; Artists; Artisans; Embossing; Stephen Benton; MIT Media Lab

\section{Introduction}

Practitioners are often the principal narrators of the evolution of a young technical subject, and the first judges of its significance and potential. Within such communities

Sean Johnston is Senior Lecturer in Science Studies at the University of Glasgow. Correspondence to Sean F. Johnston, University of Glasgow, Crichton Campus, Dumfries DG1 4ZL, UK.

E-mail: S.Johnston@crichton.gla.ac.uk 
of technologists and scientists, while the validity of technological progressivism has been eroded over recent decades, it has continued to inform judgments of success. Historians of science and technology can inadvertently sustain such viewpoints by omission, overlooking subjects that do not demonstrate commonly recognized indicators of achievement. ${ }^{1}$ Such criteria are usually taken to include the intellectual, cultural and economic impact of new sciences and technologies. Other, sociological, indicators may include the emergence of a disciplinary presence in academic curricula; a professional identity; and, the growth of occupations related to the new subject. Yet the absence of some of these characteristics excludes a wide range of subjects in science and technology from consideration, and indeed some historians and sociologists argue that such fields represent a distinct class. ${ }^{2}$ These unstable subjects resist recent sociological explanations of consensus. They may not, for example, show convincing closure of technical and intellectual debates. ${ }^{3}$

Further attention is needed to clarify how interest groups can differently assess 'success' and 'failure,' and thereby influence the fate of technologies and their subsequent historical evaluation, but a more sensitive approach than studying these two alternate end-points is to study attributions of progress. During the evolution and lifetime of a technology, outright success and failure are seldom judged; instead, practitioners and adopters evaluate progress so as to apply corrective measures, make decisions about adoption or revise forecasts. Only in retrospect does the subject acquire the totalizing label 'success' or 'failure.' By observing how progress is evaluated group by group and case by case, we can gain a clearer understanding of their effects on the technological trajectory and ultimate judgment of a subject, and how they relate to historians' own assessments. Such analysis may reveal the overgeneralizations and unbalanced perspectives that can promote faith in technological determinism.

A study of this kind is perhaps easiest for a young subject that has attracted several technical constituencies and for which consensus has been elusive. Such a subject is holography, the science of three-dimensional imaging based on optical interference. From its conception in 1947, the field has repeatedly mutated and successively enrolled new communities of practitioners, sponsors and consumers. While in some respects a typical post-World War II technical subject, holography has been unusually wideranging in the applications and social groups that it embraced. The subject found relatively stable niches as a scientific specialty and technical solution, but has attracted ambivalent assessments of progress. Holography has been both vaunted and criticized based on contrasting criteria of an unusually broad range of technical communities. As a result, it is a rich historical case for exploring attributions of progress, success and failure.

How do the backgrounds of different communities, and changing scientific, economic and political environments, influence the reception of a new technology? This paper examines how notions of success and failure became linked with expansion and progress. Holography illuminates the peculiar post-World War II environment that melded the military, commercial and popular engagement with scientific and technological subjects, and the competing criteria by which they assessed their products. 


\section{Origins and Forecasts}

During the first two decades of holography (1947-66) concepts coalesced but forecasts shifted dramatically. The nascent subject was shaped in three intellectual environments, and became linked to existing concepts, inventions and metaphors, each of which shaped perceptions of its prospects and defined its criteria of success.

Holography began as a technology founded on a novel scientific insight. Dennis Gabor (1900-79), who conceived what he called 'wavefront reconstruction' while working as an engineer at British Thomson-Houston in Rugby, England in 1947, described it as an invention for improving electron microscopy. He envisaged his twostep imaging technique as a method of avoiding the inherent aberrations of electron microscope lenses.

The key idea relied on optical interference using monochromatic light. A wavefront of light falling on an object is perturbed by it-diffracted around opaque edges, delayed in being transmitted through transparent portions, or otherwise modified upon reflection. If the light source is coherent (that is, of a well-defined wavelength and having stable phase), it will form a pattern of fine fringes if the perturbed and unperturbed portions of the optical wavefront are brought together: the resulting dark and bright regions map destructive and constructive interference between the two portions. Gabor's insight was that this optical interference pattern, which he dubbed a 'hologram,' could be used subsequently to reconstruct a faithful three-dimensional image of the original object when illuminated by coherent light.

According to Gabor's implementation, the electron beam of an electron microscope could be used to produce such a hologram of a microscopic sample. Being coherent owing to its well-defined energy and origin from a small aperture, the electron beam would be diffracted by the microscopic sample to form a 'physical shadow,' or interference pattern, on photographic film. Once developed, the hologram would be placed in an 'optical synthetiser' (sic) to use its diffraction pattern to reconstruct an enlarged and complete image, which could be corrected optically to overcome the limitations of the electron lenses used to record it.

Based partly on the positive response to the concept from scientists such as Sir Lawrence Bragg, Max Born and Sir Charles Darwin (grandson of the evolutionist, and Director of the National Physical Laboratory in Britain), Gabor joined Imperial College, London as a senior academic in 1949, continuing development of what he called 'my favourite baby' via an industrial research grant with colleagues at Associated Electrical Industries (AEI). ${ }^{4}$ Nevertheless, Gabor, who by then had over twenty years' experience as an inventor in areas as diverse as gas discharge lamps, stereoscopic cinema and speech compression, conceived wavefront reconstruction in narrow terms. He sought a patent framed in terms of apparatus for microscopy, predicting that the tool could be developed to reveal individual molecules. ${ }^{5}$ A handful of other investigators pursued Gabor's ideas, usually starting from the standpoint of microscopic imaging using X-rays, electron beams or radio waves. ${ }^{6}$

Given Gabor's identification of the technique with microscopy, and improved optical resolution as a criterion of success, his technique had a restricted life. By the late 1950s 
he and his collaborators had abandoned their research owing to a variety of difficulties. The AEI workers, attempting to alter their commercial microscope to record interference fringes, blamed technical limitations. They found that their electron source was not adequately coherent, ${ }^{7}$ and vibration, sample heating and instability of the electron beam further aggravated their problems, but Gabor also attributed social factors to the affair, blaming wavering commitment from his industrial colleagues and conservatism of microscope manufacturers. Ascribing their 'failure' to inadequate motivation, and concerned that publicity would sour researchers to the subject, he complained to the Research Director at AEI, T. E. Allibone, that he did 'not like being the bright boy who produces brilliant dud ideas. ${ }^{8}$

Nevertheless, Gabor and his student W. P. Goss experienced their own technical difficulties in developing the optical synthetizer stage of the apparatus. The principal problem was a 'conjugate,' or undesired twin, image reconstructed from the hologram. This fuzzy and inextricable second image overlapped the desired reconstruction, rendering the technique unsatisfactory for practical use. Gabor struggled to devise optical methods of subtracting the twin image to improve image quality, but found that his carefully fabricated optical schemes demanded extremely high mechanical tolerances and precise optical alignments to yield even mediocre results. Other workers seeking a theoretical solution to the twin-image problem also found themselves facing dead ends. By 1956, the most enthusiastic of them, Gordon Rogers, wrote privately, 'As far I am concerned, I am quite happy to let Diffraction Microscopy die a natural death. I see relatively little future for it, and am looking forward to doing something else. ${ }^{\prime 9}$ And two years later, Allibone publicly narrated the work in historical terms, dismissing it as an unproductive detour for his company that had, at least, led to further work in correcting the shortcomings of conventional electron microscopes. ${ }^{10}$

From the perspective of practicing microscopists, Gabor's technique was judged to be unpromising and arcane, a conclusion exacerbated by his limited practical achievements in attaining his own research targets. Moreover, by merging electron microscopy with visible optics, wavefront reconstruction had aspects that appeared retrograde rather than progressive. For instance, instead of the immediacy of seeing an image on a fluorescent screen (as some electron microscopes then produced), the reconstructed image was to be obtained more painstakingly via a 30-minute exposure, conventional photographic processing, unintuitive optical transformation, and observation through a conventional microscope eyepiece. ${ }^{11}$

In their various ways, these evaluations were shaped by their investigators' backgrounds and working contexts, and judged Gabor's wavefront reconstruction distinctively during its shaky 10 -year run. The 'failed' subject nevertheless was rehabilitated posthumously; indeed, Gabor was to be awarded the Nobel Prize in Physics for holography in 1971. Unlike early accounts that stressed unsustainable progress, later stories could tell a tale of triumph over adversity; of tenacious and even heroic workers toiling in obscurity; of a chain of insights leading to a general principle and patent claims; and of the inevitable success and progress of science.

This overturned judgment followed American research pursued quite independently of Gabor and his contemporaries. A research engineer at an American classified lab, 
Emmett Leith (b. 1927) of the Willow Run Laboratories (WRL) of the University of Michigan, had begun work in 1954 on the processing of signals from a new form of radar, known as synthetic aperture radar (SAR). He and colleagues at Willow Run had devised a means of using coherent light to transform the radar signals, recorded in the form of gray tracks on a long strip of film, into a high-resolution optical image.

By 1956, Leith had conceived a relationship between this process and the theory of physical optics. In 1960, Leith and his colleague in the Radar \& Optics Lab, Juris Upatnieks (b. 1936), began to extend Gabor's work from their distinct cognitive standpoint of optical information processing, a fertile hybrid of communication theory (familiar to the electrical engineers that dominated WRL) and physical optics (familiar to physicists). They developed a technique that sidestepped the technical disadvantages of the twin image problem. ${ }^{12}$ Impressive results followed from this research: first, the ability to produce clean reconstructed images of line drawings in early 1961 ; $^{13}$ second, high-quality grayscale images at the end of 1962; and finally, with the use of the newly-available laser as a coherent light source in late 1963, an astonishing form of three-dimensional imagery in which the reconstructed images exhibited depth and parallax with unprecedented realism. In May 1964, Leith's and Upatnieks' three-dimensional hologram was announced and demonstrated at the Spring Optical Society of America meeting, and over the following months conference papers, newspaper reports and press releases portrayed it as 'lensless threedimensional photography.' ${ }^{14}$

The accounts nevertheless strained to represent the perceived link with the photograph. The Leith-Upatnieks hologram was a kind of transparency, but the image was observed by looking through the hologram as through a window. Its featureless surface was described as storing the image for later reconstitution. The copy of a hologram yielded not a negative image, but another positive. And unlike a photograph, the hologram could recreate a view of the entire image from any part; the pieces of a broken hologram still worked. The technique was also restrictive: only small laboratory scenes could be recorded. The transmission hologram was tied to the laser as a light source, not just for its initial recording but also for subsequent reconstruction. The unfamiliar attributes of this 'window with a memory' were difficult to reconcile with concepts of photography but, despite the imperfect correspondence, photography was to be a convenient guide to understanding the new medium and in forecasting its future development. $^{15}$

In parallel with these American developments but equally hidden in its early stages was work pursued by Yury Denisyuk (b. 1927) in Leningrad. Denisyuk, an optical researcher at the Vavilov State Optical Institute since 1954 and beginning an advanced degree there four years later, was investigating means of recording the wavefront of light reflected from an object. He extended ideas that had been pursued at the turn of the century by Gabriel Lippmann, who had conceived a technique for recording the interference of light through the depth of a fine photographic emulsion. When Lippmann's special recordings were subsequently illuminated, they could reconstruct the full color of a scene by constructively reinforcing the component wavelengths that had recorded the regular layers. Denisyuk considered an extension of the same process using coherent 
light, and deduced that such interference layers captured not only the wavelengths used, but also the geometry of the wavefront of light itself. His 'wave photographs' could reconstruct the image of a three-dimensional surface, but without the necessity of a focusing lens. Using a mercury arc light source, as had Gabor, Leith and Upatnieks, Denisyuk created three-dimensional images of curved mirrors and simple reflective objects. ${ }^{16}$ The technique, subsequently known as 'Denisyuk,' 'Lippmann' or simply 'reflection' holography, was different in concept and implementation from those of Gabor and Leith and Upatnieks. It reconstructed three-dimensional images by reflection from the hologram in normal ('white') light, and had no link with either microscopy or image processing. Instead, its more demanding recording conditions and viewing arrangements made it rather analogous to a 19th century daguerreotype, but with the addition of depth and (potentially) color. ${ }^{17}$ While Denisyuk initially struggled to conceive applications of his concept, he portrayed his technique as a superior form of photography for a limited class of objects, or as a color-dependent optical element. Because of this unimaginative portrayal and lack of an influential mentor, his Soviet contemporaries largely ignored Denisyuk's research.

Thus the work of Gabor, Leith-Upatnieks and Denisyuk created at least three versions of an intellectual concept and its associated technologies: either an instrument for improved microscopy; a type of three-dimensional, lensless photograph in the form of a transmissive window; or, a method of recording the complete optical properties of a shallow object on a reflective plate. These divergent conceptions, arising from different technical and occupational contexts, profoundly shaped the early forecasts of the subject known by 1966 as holography. Moreover, their respective 'successes' were differently evaluated. Gabor's narrow portrayal of wavefront reconstruction during the 1950s had yielded few forecasts beyond improved microscopy. His concept was selflimiting and of interest principally to workers interested in ultra-microscopy and the then limited field of physical optics. Denisyuk's self-assessment was similarly derided or ignored. By contrast, the Leith-Upatnieks conception excited great interest far beyond the domain of physicists and engineers. The intellectual locus for these initial forecasts was photography, and the geographical locus was the town of Ann Arbor, where the University of Michigan and the Willow Run Laboratories were based.

\section{The Conductron Corporation and Commercial Predictions of Holography}

For most American observers, the Leith-Upatnieks technique was framed in terms of a potential success story. Popular accounts portrayed the viewing of a hologram as a sublime experience filled with childlike wonder, as an outgrowth of photography, as an expression of modern science, and as an illustration of industrial and scientific collaboration.

Awe was an emotion commonly voiced by viewers of holograms. The notion of a 'technological sublime,' explored by historian of technology David Nye, is relevant to the experience. ${ }^{18}$ While Nye focuses on the effects of large-scale technology in evoking a sense of the sublime (immense dams, complex railways and the atomic bomb, for example) I would argue that holograms and their viewing environments had a 
profound impact akin to glimpsing a rare natural phenomenon. Consider the rainbow appearing after a severe storm, and dividing the sky into bright and dark regions by its colourful arc; ice haloes appearing in the shrouding fog of a cold morning; bright lightning bolts in an otherwise inky sky; the heiligenschein, a halo of light surrounding the shadow of one's head reflected from a field of dewy grass; or the glory, a circular rainbow around the shadow of an airplane as it flies above a cloud. Each of these optical phenomena is experienced in a peculiar context that isolates the observer from the everyday world. ${ }^{19}$

The same transcendent experience could be experienced in demonstrations of holograms. Like natural phenomena, the experience of viewing a hologram awed early observers because of the profoundly unfamiliar imagery and disorienting environment. The holographic images of the 1960s were isolated from the viewer, appearing behind the hologram surface, and were illuminated by the unnatural speckled light of the laser. Rooms were dark, and sometimes shrouded in curtains; the hologram was illuminated by a hidden source, appearing bright and disembodied from its environment, and most importantly, the image was three-dimensional, realistic and yet untouchable. Even when, like the first generation of holograms made by scientists, the images were mundane-tools, trains or chessmen — the context gave them an aura of ghost-like unfamiliarity.

The viewing experience suggested hidden potential to early observers. Holography as a subject seemed replete with latent possibilities for new imaging applications, and there were veiled uses, too: the Willow Run Laboratories, having the largest early concentration of holographers, continued to focus on classified applications of optical data processing for its sponsors in the Department of Defense. One consequence of the parallel public and hidden lines of research was that Leith's and Upatnieks' contributions were initially eclipsed by a new colleague in the more academically open Electrical Engineering Department of the University of Michigan. ${ }^{20}$

Nevertheless, early commercial expansion of the technology went hand-in-hand with military sponsorship. The most significant early explorations of holography's commercial potential were made at the Conductron Corporation in Ann Arbor. The company had been founded in 1960 by Keeve M. (Kip) Siegel, an engineer-entrepreneur who had joined the Willow Run Labs in 1948 and heading its Upper Atmospheric Physics Group between 1949 and $1953 .{ }^{21}$ Siegel, a larger-than-life figure who directed the laboratory authoritatively but charismatically, populated his new Conductron Corporation with employees drawn from WRL and supported initially, like Willow Run itself, by military contracts. $^{22}$

The Conductron Corporation took up holography by the same route that Leith had been drawn to it-via synthetic aperture radar (SAR), and using surplus resources from lucrative development contracts. One of their first contracts, directed by physicist Gary Cochran, was to produce optical processing equipment for SAR data processing. By late 1964, he had made his first hologram, and later recalled:

Kip Siegel had a chance to see it, and he was fascinated. Kip Siegel was a promoter. He was the kind of guy [who felt that] it didn't matter whether you made any money if he could get the excitement of Wall Street bankers and get more investment capital. He saw the 
potential .... Siegel was clearly interested in developing the technology as a tool of investment. $^{23}$

As Cochran began to study the field, making bigger and better holograms, Siegel became seduced by the technology. From 1965, he dedicated Conductron's optical team to pursuing impressive and sublime applications of holography.

Siegel proselytized a merging of military and commercial domains. Underlying it was strong faith correlating the advancement of knowledge with inevitable technological impact. Conductron's growing variety of display pieces was intended to illustrate inexorable progress: in effect, the company attempted to suggest determinism by boot-strapping commercial interest, attracting investment financing to further develop technical improvements. By early 1967, Cochran's 40-strong optics group was responsible both for SAR contracts for the military and for hologram production and development aimed at would-be corporate sponsors.

They worked initially to advance the technical possibilities of display holograms, moving from $4 \times 5$ inch to $8 \times 10$ inch and then $11 \times 14$ inch 'window' holograms during 1966. Holograms, lit by filtered mercury lamps, were increasingly produced for trade show displays. More dramatic but less practical holograms were shown only to visitors in the Conductron lobby or lab. For instance, when a helium-neon (red) and argon (blue and green) lasers were combined to make separate holographic exposures on the same hologram plate, they could create a color image. While such holograms attracted interest and exemplified the technical progress being made in image reproduction, their display requirements (two carefully aligned and power-hungry lasers) made them too unwieldy to be sold or even displayed outside the lab. The notion of progress was thus problematic from the outset: displays became more impressive, but the necessary equipment multiplied in cost, complexity and unreliability.

Progress was also touted in terms of production range and capacity. Between 1965 and 1970, the firm created over a thousand custom holograms for clients ranging from Hoffman La Roche (pharmaceutical displays for trade shows) to General Motors (lobby, museum and trade show displays) to artists (Richard Wilt and Bruce Nauman). Conductron's most important customer proved to be the publishers of the World Book Encyclopedia, who contracted the company to produce holograms to be included in the forthcoming 1967 Science Year. The project required the copying a 'master' hologram of chess pieces to make some 500,000 copies on film. The resulting copies, bound into the books, could be illuminated by a flashlight directed through a supplied red plastic filter. ${ }^{24}$ This unprecedented achievement nevertheless provided ambivalent commercial success: production costs were covered, and the publication proved the best-selling edition of the Science Year, but no orders of comparable size followed. ${ }^{25}$

Beyond such status-raising achievements, Siegel directed Conductron's staff to develop dramatic demonstration pieces to support his predictions for holography. What had been identified as an autonomous technology became ever more intentional and stage-managed. A consummate showman, Siegel's unrestrained forecasts had a strong effect on public expectations. A typical example of Siegel's rhetoric was the claim that, by 1975, 'the United States will have, as far as new products are concerned, only three-dimensional television and three-dimensional movies on the market. I would not 
expect two-dimensional processing, two-dimensional television, two-dimensional home movies to continue-that's my personal belief. I don't think people will buy things that are antiquated.'26

One of Siegel's most public predictions was the inevitable development of holography as a medium for recording large-scale events, epitomized by his goal of making a holographic movie of the Olympic games. When his new McDonnell Douglas partners remained skeptical of the feasibility of holographic movies, Siegel's engineers devised a holographic animation to demonstrate the feasibility of such applications. Using a loop of $70-\mathrm{mm}$ wide film, they recorded several dozen individual hologram frames of a model merry-go-around, repositioned manually for each individual holographic exposure. For reconstruction, the engineers adapted a commercial projector to illuminate the frames successively in a continuous loop. The result was an animated (and cyclic) 3D movie. The reconstructed scene was viewed through magnifying optics to give a realistic perception of a three-dimensional changing scene.

Despite their severe technical constraints, the short animations were portrayed as a commercially important step towards holographic movies and television. This approach of producing a proof-of-concept model was an extension of methods pursued in military research contracts. Siegel's company sought to continue this feedback cycle (attracting more investment capital for ever-more impressive demonstrations) to the point of yielding viable commercial products. Such activities also encouraged optimistic research for solutions to unlikely goals, in a self-fulfilling prophecy. For example, while the rather limited demonstration device illustrated the principle, Conductron engineers realized that producing practical holographic movies would require a highpowered pulsed laser to record moving objects—-such as people-before they could move enough to smear the interference fringes on the photosensitive plate, and that such a laser for recording outdoor scenes would certainly have dangerous and probably unattainable power requirements. ${ }^{27}$ Nevertheless, during 1966 and 1967, they developed a pulsed ruby laser that produced a sufficiently intense beam to record first a human hand, and soon a human portrait in a darkened room. ${ }^{28}$ When further technical improvement proved impossible, the Conductron marketing staff sought to redefine the goals. They turned from holographic movies to three-dimensional human scenes, recording holograms of commercial displays, groups of poker players and (perhaps apocryphally) nude dancers. ${ }^{29}$

Siegel's impossibly optimistic predictions and speculative funding sustained the momentum at Conductron until 1967, when he resigned to form a new start-up firm, KMS Industries. The design of dramatic demonstrations and the pursuit of paying customers for holograms continued at both Conductron and KMS Industries for a further three years. When McDonnell Douglas moved the Conductron operation to Missouri in 1970, it even more intensively marketed the commercial possibilities of pulsed holography for advertising purposes. ${ }^{30}$ At his new company on the other side of Ann Arbor, Siegel created a new holography team to pursue viable applications and further enticements for investors.

The 'success' of the Conductron development was thus ambivalent. The pulsed-laser activities were company- and investor-funded and commercially unproductive, but 
illustrated clear technical progress in a limited domain over a short period. Through such developments, the company became the messenger of a particular view of progress. For Conductron, the hologram was an effective icon, embodying all that was new and valued in that well-funded decade, and melding the laser, high science and awe-inspiring imagery into an example of seemingly inevitable technical advance. ${ }^{31}$ This divergence between marketplace reality and commercial claims is not unique, of course: it was a feature in other new technological fields such as nuclear power during the 1950s, biotechnology from the 1980s and nanotechnology from the 1990s. ${ }^{32}$ Even the vocabulary associated with such developments can unconsciously suggest that improvement is somehow inherent, via phrases such as 'emerging technology' or 'promises outpacing achievements'.

In a more restrained fashion, other firms, too, cited practical indicators of progress for the new science. From the late 1960s, for instance, 'holographic interferometry' or 'holographic non-destructive testing' (HNDT), a sensitive technique for measuring mechanical motion or deformation, became popular with metrologists and mechanical engineers and found a market niche. A commercial holographic tire-tester developed by the Ann Arbor company GC-Optronics, for example, allowed the lamination of airplane tires to be verified rapidly. For this application, the criteria of success were economic (lower costs) technical (better testing reliability) and social (improved customer safety).

Nevertheless, these criteria were not often attained. By 1970, Clark Charnetski, a physicist at Conductron, could summarize what the company had already learned in its own attempts to promote holography. He noted that the spectacular predictions made by Siegel and others had provided large investment capital but few commercially viable applications. To the surprise of Conductron engineers, display holography had been less important economically than had other uses such as holographic nondestructive testing. Charnetski ascribed this to the cost and difficulty of producing holograms of large scenes and in developing copying processes. But the problem also had non-technical dimensions:

Holography was and still is largely done by scientists and engineers, and these people are as a whole more interested in the scientific things and therefore lend their talents more readily to scientific applications than toward breaking into the advertising game. ${ }^{33}$

The often-vain exploration for potential markets led a number of large firms to withdraw quietly from the field by the early 1970s. For instance, CBS Laboratories employed Dennis Gabor as a consultant but developed no promising products. RCA developed a prototype consumer video playback system (Selectavision Holotape) in 1969, but canceled the project in 1972 when the firm was in financial difficulties and facing competition from more versatile magnetic recording technologies. McDonnell Douglas closed its pulsed holography operation in 1973 owing to inadequate interest from the advertising industry and corporate customers. IBM's early enthusiasm for holographic computer memories did not culminate in products, and Polaroid Corporation, while developing recording techniques and new photosensitive media during the 1970s, did not effectively market them. 
The enthusiasms of technologists did not necessarily translate to those of wider culture. Marketing holograms proved unexpectedly difficult, and there was increasing disjunction between technical forecasts and economic reality.

\section{Sponsorship and Community Values}

The Conductron experience illustrates how one community-a nascent commercial group operating according to practices absorbed from military-contract researchdefined holography and its accomplishments during the 1960s. Its engineers were habituated to exploring applications creatively in a classified context and with relatively abundant funding, and were imbued with confidence in the very notion of progress. They arguably were less sensitive to commercial pressures than were typical workers in industry, however, because they envisaged holography as a dramatic and inevitable extension of photography. Conductron consequently sought display applications that highlighted its three-dimensionality and visual impact. Less convincingly, its engineers forecast and pursued the extension of holography to color imagery, movies and television based on the technical trajectory of those earlier imaging media, and most misleadingly, again using the analogy of early photography, they predicted a rising public appeal and inevitably growing market just like its antecedents. ${ }^{34}$

Ann Arbor, while generating the first cohort of holographic researchers, had an atypical context of research and funding. Other academic scientists and commercial engineers, less dependent on military sponsorship, operated in different intellectual, funding and social environments. Moreover, by 1970 a wider range of adopters was becoming involved in the subject, particularly artisans and artists inspired by the counterculture and technological art, respectively. ${ }^{35}$ Each of these embryonic communities developed a unique perspective on the nature of the subject, its goals and criteria of success.

As discussed above, for military sponsors and the scientific holographers who pursued research for them, the demonstration of theoretical extensions and technological potential were paramount. Military supremacy required a competitive edge, and sponsors favored bold replacements of existing technologies. The result created an expectation of continual extension of knowledge and technical capabilities, and a continuance of the relative freedom from economic concerns that had dominated military research since World War II. There was a consequent faith in both positivism (particularly in its view of knowledge as incrementally and inexorably growing) and technological progressivism (inevitable improvement in performance measured according to self-evident technical criteria), with both assuming social improvement as a foreseeable by-product.

For artists, on the other hand, success in holography was evaluated according to different criteria. The medium had to have adequate technical versatility to support aesthetic expression, and the new art form required the acceptance of art critics and a receptive public. While this was relatively free of positivist underpinnings, these criteria did embody implicitly progressive ideas, namely the assumption that the capabilities and audience for the medium would expand. 
Different criteria applied to artisans who took up holography during the early 1970s. Their definition of progress was a combination of growing audiences and income from the sale of holograms, alongside the development of new techniques of production, especially cost-effective or simplified methods. But, as with other communities of holographers, success was defined in terms of expansion, which amounted to anticipation of continual progressive increase. These practitioners supported their work by operating schools of holography in non-academic and non-scientific contexts, through custom work for clients, and by the sale of holograms to the public.

It is noteworthy that the community of artisans, like aesthetic or fine-art holographers, often supported this implicit assumption of technical progress and consequent mass popularity even while embracing countercultural themes. Thus for all three communities - scientists, artists and artisans_-stagnant conditions, measured in terms of income generation, technical abilities, and acceptance by critics, consumers, students or the wider public, equated to failure for the subject. ${ }^{36}$

During the 1980s, despite its exposure to hundreds of thousands of viewers through public exhibitions of holograms, and the growing ubiquity of mass-produced holograms, the subject could not be characterized reliably by these criteria, and appeared different to each constituency. The indefinable state of progress can be illustrated by bibliometric indicators: by the mid 1990s, while the annual publication rates of papers and patents were rising, those of books and theses were falling, and the number of scientific conferences and hologram art exhibitions had diminished to half their value of a decade earlier. ${ }^{37}$ For artists and artisans, the field was declining; for scientists, it had periodic ups and downs; but for inventors and investors, it continued to look promising. Economic, rather than population, indicators consequently became a widely accepted mark of success of the medium. For different communities then, holography was a subject that either evinced obvious success, remained latent with potential, or had outlived its promise.

\section{Segregating Communities: Judgments of Successful Imagery}

The contrasting judgment of separate constituencies is further illustrated by the goals of holographic imaging. The emerging occupational specialists of holography variously identified the strengths and weaknesses of holographic technology. They argued that a collection of limitations surrounding the hologram prevented the expansion of the technology of holography in wider culture. This was a two-way process: their mutually incompatible criteria encouraged the holographic communities to differentiate further. The divergence of holograms and their associated communities is consistent with the framework of social construction of technology (SCOT) and its concepts of relevant social groups and interpretative flexibility. ${ }^{38}$ However, for most of these groups consensus and closure were not attained. Instead, their technical goals diverged while they continued to seek improvement for their medium, making stability elusive.

The gaze of these distinct communities identified dissimilar limitations for the medium. The first to be noted were problems with the laser itself. ${ }^{39}$ Holograms illuminated by lasers revealed the phenomenon of laser speckle. The reconstructed 
image and, indeed, any surface illuminated by the laser, appears to shimmer and sparkle with a graininess that depends critically on the position of the observer's eyes. The effect, resulting from the random constructive and destructive interference from the highly coherent laser light (having a narrow distribution of wavelengths and light waves that were 'in step,' producing interference over centimeters or even meters), was exacerbated by diffusely reflecting surfaces. This technical artifact could mask the otherwise stunning imagery of the hologram, but was a complaint of scientists and engineers more than aesthetic holographers: laser speckle was a particular problem when photographing holograms, an activity common in scientific and engineering studies, but relatively unimportant for casual viewers. ${ }^{40}$

On the other hand, the cost of the laser was a crucial constraint for artists and advertisers but of relatively little importance to well-funded scientists. Continuous Wave (CW) lasers remained prohibitively expensive for galleries to purchase in moderate numbers, and pulsed lasers were out of reach of most laboratories. Lasers were also unfamiliar and intimidating for non-scientists, and their use was curtailed severely with the introduction of safety legislation in the early 1970s concerning eye exposure to laser beams, but lasers were also relatively dim light sources for reconstructing the holographic image. Seldom exceeding tens of milliwatts of optical power, the laser was adequate to illuminate a single hologram well in a normally lit room, but not in a daylight-illuminated shop window. If any other form of light were used to reconstruct a hologram, the image would be unacceptably blurred. Neither of these restrictions was a particular problem for scientific applications such as holographic interferometry, but judged to be a severe limitation for public displays.

A third characteristic constrained holography as a medium for portraits. The very monochromaticity of laser light provided eerily unworldly images akin to the street illumination from sodium lamps, or the orthochromatic (blue- or green-sensitive) images of early photographic and cinematographic films. The contrast and tonal gradations of reconstructed images appeared unfamiliar, and was inferior to the panchromatic black-and-white films that had been used universally since World War II.

This effect was exacerbated in the first portraits made with pulsed ruby lasers. Because human skin is slightly transparent to the wavelength of a ruby laser, portraits made subjects look waxy-skinned, blotchy and disturbingly morbid. Unlike photography, its perceived analog, holography failed to develop a market for portraiture, and artists who adopted pulsed lasers more successfully employed them for figure studies rather than facial depictions. For photographers, holographic portraiture and color holography represented problems, not progress.

A further constraint was that holographic images were static, and thus of limited interest for media applications. Indeed, the imagery of living things represented an instant of time, frozen in space. Holograms produced with pulsed lasers, acting like a fast flash camera, captured unsettlingly frozen facial expressions of their subjects who had been sitting in near darkness, often accentuating the unfamiliarity by showing the unusually wide irises of the dark-adapted eye. ${ }^{41}$

In sum, these limitations restricted holography to a narrow class of subjects and to a seemingly unnatural or stylized representation, paradoxically in opposition to its 
highly realistic perspective. The 'problems' and putative 'solutions' were differently ranked for different communities, however.

The most pressing problem for aesthetic and commercial users (but irrelevant for scientists) was the need for a laser to display the hologram. Denisyuk's holograms of the early 1960s offered a solution: they produced a clear green image when viewed in sunlight or room lighting. But creating such holograms demanded extremely highresolution photographic emulsions and very stable conditions during the exposure. They became popular in the Soviet Union from the mid 1970s as a means of recording historic objects, but had a low uptake in the West because the emulsions and chemistry were not readily available, and because artists perceived them to offer limited options for creativity.

Another technical approach was the 'image plane' hologram, in which a hologram of a hologram (a 'second generation' hologram) was recorded. ${ }^{42}$ Such a hologram produced little color smearing of reconstructed images for points near the plate, so a white light source was adequate to view holograms of shallow objects. A secondary advantage was that such images were even more striking than conventional holograms: the image appeared to pass through the hologram plate. This appealing attribute became ubiquitous in commercial and art holograms by the late 1970s, but was of little interest to scientists.

During the early 1970s, the 'rainbow hologram' also became widespread. The technique, later dubbed 'white light transmission holography', was reported tangentially by Polaroid scientist Stephen Benton (1941-2003) in 1969 while his employers sought a patent, but became more widely known only from 1973 after his collaboration with artist Harriet Casdin-Silver (b. 1925). ${ }^{43}$ By employing a special optical geometry to record the hologram, a sharp image could be viewed in white light, although cast in a spectrum of colors that shifted with the viewing position. Benton became a well-known intermediary between scientific and artistic communities, particularly after his move to the new MIT Media Lab in 1984. The Media Lab, uniting a collection of enthusiastic engineers and scientists, sought to transform culture via new media technologies, aiming, as one breathless account put it, to invent the future. ${ }^{44}$ Benton's Spatial Imaging Group was funded by sponsors courted by Conductron 20 years earlier: General Motors and the American military, via the Defense Advanced Research Projects Agency (DARPA).

Rainbow holograms importantly reduced the cost of displaying holograms, but had an uneven popularity that further divided supporters. East-coast American holographers, close to Benton's Massachusetts laboratory, adopted rainbow holograms more enthusiastically than did their west coast and European counterparts. Artists championed the technique, discovering that, by overlaying several exposures, a single hologram could display multi-colored images. However, rainbow holography was rejected by Soviet practitioners, who saw the technique as complex and much poorer in quality than their own reflection holograms, and by most American scientists, who were concerned with the accurate recording and dimensional analysis of three-dimensional objects or transitory events. ${ }^{45}$

By allowing white-light reconstruction of the holographic images, these techniques removed the need for an expensive laser for viewing, and also removed the objectionable 
speckle that went with it. They were also bright (especially for rainbows), although usually limited in depth. Bleaching the photographic emulsion allowed holographic images to be even brighter, aiding the art community and the most promising market niche of the 1970s, advertising.

From the 1980s, 'embossed holograms' provided new opportunities for technical judgments and forecasts. Embossed holograms combined image-plane and rainbow holography in a reflective form that could be reproduced using adapted printing-press technology. Manufactured by the millions on metal foil, they became ubiquitous in packaging, graphic arts and security applications. ${ }^{46}$ While this brought holograms to a much wider audience, it generated dramatically divergent judgments. Unlike the previous varieties of holograms, this new type generated not just indifference from different communities, but outright animosity.

Embossed holograms were inexpensive, reducing the cost of copies by a hundredfold. They could be mass-produced reliably using a number of proprietary techniques that seemed less constricted by patents than were earlier products. They were chemically and mechanically stable, unlike most previous hologram materials that were susceptible to scratches, humidity or aging. Together, these technical advantages promoted the widespread application of embossed holograms.

However, connoisseurs of imaging — the self-styled 'display holographers' made up of artists and artisans-derided embossed holograms. The flexible backing of embossed holograms, particularly those on magazine covers, caused color shifts and image distortion, and because the holograms were usually viewed in uncontrolled lighting, images could appear fuzzy or dim. In response to these limitations, their producers progressively simplified the imagery to incorporate shallow, eye-catching patterns, a product that some in the industry contemptuously dubbed 'shiny shit. ${ }^{47}$

By moving toward less ambitious images, embossed holograms evolved to minimize their perceived weaknesses and to exploit new markets. While applications such as magazine illustrations declined, others expanded, and created new industries and adopters. Visual appeal was redefined. Their image characteristics made embossed holograms particularly suitable for attention-grabbing product packaging (a profitable and growing industry from the early 1990s) and for security applications, where any defect in the complex pattern could indicate tampering or counterfeiting. ${ }^{48}$

This technical mutation, while capturing a large market and the first undeniably profitable application of holography, arguably amounted to a reversal of the original aims of the medium. Yet consensus about success could not be defined in utilitarian terms. Embossed holograms promoted low-cost mass production but had relatively poor image quality; they brought three-dimensional imagery to vastly increased audiences, but simultaneously reduced the sublime characteristics of depth, parallax and image clarity. Security applications exploited the complex color shifts and angledependence of embossed holograms, making the forgery of credit cards and bank notes more difficult, but for imaging purposes, these characteristics were deemed to be a serious defect. Fine-art holograms became less popular, with artists complaining that embossed holograms irreparably devalued the aesthetic attraction of the medium. ${ }^{49}$ This expansion of holography into the mass market was thus judged by its initial 
supporters to be a failure, because it had deviated from their forecast trajectory. The 'shiny shit' had defiled the utopian predictions.

The evolving techniques for producing bright, white-light holograms thus both liberated the growing field of display holography in the 1970s for commercial use and constrained its acceptance in the 1980s, particularly for artists. The tribulations of display holographers were not faced by most scientific and engineering users, who continued to employ laser-viewable holograms; nor were they recognized by marketers of packaging and anti-counterfeiting holograms. Thus, the applications of holograms supported the growing segregation of practitioners and conflicting definitions of success.

\section{Wider Judgments: Critics and Consumers}

Gabor's wavefront reconstruction, Conductron's exploration of commercial markets, and the ambivalent assessments of display holography illustrate the varied judgments attached to the subject. For all communities of holographic practitioners, however, expectations of progress remained strong. Indeed, some forecasts, oft rejuvenated, seemed impervious to attributions of failure despite the continued lack of demonstrable viability. Among these were predictions of holographic 'optical memories' for retrieving data. ${ }^{50}$ One reason for the endurance of such promised applications was faith in technological progressivism and determinism by its advocates, even when uncorroborated by evidence. It was expected that the new invention would not only provide new and better things, but would necessarily change culture and wider society as a result.

Nevertheless, holography was repeatedly cited as having failed in expanding enthusiasm, garnering audiences and developing markets—a failure, in effect, to conform to the expectations of technological progressivism. Artists responded with dismay, for instance, to negative reviews of a large hologram exhibition, Holography' 75 , held at the International Center for Photography in New York, which curtailed their expectations of aesthetic acceptance and growing markets. ${ }^{51}$ Both critics and artists (to their chagrin) portrayed holography as immature and in a state of early aesthetic and technical development; both, indeed, were imbued with a similar definition of progress.

In order to sustain continued confidence, predictions mutated. Forecasts during holography's first active decade_-extending from 1965 to 1974 - had been uniformly expansionist, making unrealistically optimistic extrapolations based on laboratory demonstrations or even speculative applications. ${ }^{52}$ Some commentators had attributed deviations from commercial forecasts to the complexity of patent litigation, and the resulting hesitancy of many firms to employ holograms as a result. ${ }^{53}$ When, after one decade, two decades or a quarter century, material achievements were not obvious to all, the original commentators and others-notably Stephen Benton, who became prominent as conference organiser and holography pundit-recast the development of their field as an historical narrative either still linked with latent progress ${ }^{54}$ or portrayed simplistically as a classic tale of market failure. ${ }^{55}$

Yet none of these later depictions dominated public consciousness of holography. Instead, understandings became shaped by fictional portrayals, causing a bifurcation 
between the technical realities and the imagined future of the medium. These positivistic depictions of progress in fictional holography originated from the scientific and commercial forecasts of the 1960s but dramatically extended and diverged from them. For the general public, the notion of holograms as awesome but intangible images was fostered by the film Star Wars (written 1973-7, released 1977), and on television by the British comedy series Red Dwarf (pilot written in 1983, series broadcast 1988-94) and the Star Trek television series The Next Generation (broadcast 1987-94) with its 'holodeck,' or holographic visualization room, by Deep Space Nine (1993-99) with 'holosuites,' or commercially-operated holographic environments, and by Voyager (1995-2001) with its 'Emergency Medical Hologram,' or virtual doctor. These science fictional portrayals transformed the hologram from a glass plate into a more dramatic creation, ranging from mere optical playback of a recording (in Star Wars) to computer-generated lifelike characters (in Red Dwarf) to entire interactive environments that include sensations of touch, sound and smell (in the later Star Trek series). ${ }^{56}$ Popular culture thus became diverted by a virtual image of the subject that could not be realized by any community of holographers. ${ }^{57}$ Anticipation, supported by faith in progress, threatened to outstrip reality.

This splitting of real and imagined futures, evident in the earlier commercial forecasts as well as later science fictional accounts, is a theme common to many new technologies. It has parallels with the account that Colin Milburn has given of nanotechnology, for instance. Milburn argues that popular and professional writing about nanotechnology amounts to a 'teleological narrative' that transforms a dream into something that is inevitable. ${ }^{58} \mathrm{He}$ suggests that promotion of the subject has transgressed a line between 'speculative science' (an extrapolation of current scientific thinking, describing what could be) and 'fictional science' (an account of what, inevitably, will be, in some world to come). In a similar vein, Kip Siegel's forecasts could be characterized as fictional science that influenced science fiction writers a decade later. Such incredible extrapolations may not require the disorienting qualities of the hologram, though. The near-utopian predictions for holography, promoted by its fantastic early commercial claims, have been made of other, more mundane, technologies. ${ }^{59}$

In any case, such fictional diversions increased expectations, and adversely affected the cottage industries of holography that appeared during the 1980s. Small firms selling holograms for home viewing, which had sprung up in major cities after major exhibitions, failed to thrive. Small commercial galleries, such as the Holos Gallery in San Francisco, gradually discovered that sales of holograms could sustain them only if their businesses were transformed into wholesaling operations for distributing holographic trinkets to museums of science and technology. ${ }^{60}$ Popular interest and markets proved fickle, however, and few companies became profitable for long. The sale of holographic art, always marginal, declined as holographic kitsch in the form of embossed foils for children's stickers and magazine covers began to flood the market from the mid 1980s. As discussed above, it is significant that artisanal and artistic holographers identified this trajectory as non-progressive and hence an indicator of failure. They commonly characterized the altered focus of public interest as a descent similar to the history of earlier three-dimensional media, transforming them from a sublime technological 
experience to mere children's products having lower intrinsic value. ${ }^{61}$ Thus they judged the type of audience to be more important than its size.

The limited public acceptance of commercial holograms meant that real-world holographers continued to struggle for occupational status and acceptance of their products. The subject, its communities and their aspirations of progress were closely interlinked. The technical groups associated with holography proved unstable partly because public engagement and employment were themselves uncertain. Holography did not develop applications that generated a stable occupation supported by universitytaught courses. ${ }^{62}$ The growth of long-lived occupations and accredited teaching programs, usually deemed crucial for the consolidation of a new profession and a new discipline, could not be sustained by the applications of holography. Instead, the subject spawned several marginal constituencies, along with distinct forecasts and criteria of success. Even the best supported of these, the broad field of optical engineering and scientific holography, found its military and corporate funding difficult to sustain after the Cold War. Artists and artisans found their exhibitions and income reduced by the expansion of embossed holograms and changing public expectations. Colleagues in other fields consequently interpreted the relative social invisibility of holographers as a failure of the subject.

During the late 1980s, when holography was at a peak of visibility, practicing display holographers comprised an active community of about a thousand individuals ranging from scientists, to artisans, artists and entrepreneurs. The New York Museum of Holography kept files on some 280 individual holographers, and attracted some 50,000 visitors to view their products every year. ${ }^{63}$ Yet the Museum, founded in 1976 to serve not just the disparate subcultures of holography but also the general public, discovered that holographers' sense of community was ephemeral and inward looking. As discovered by the schools and cottage industry that appeared during the 1970s and early 1980s, the Museum found that the general public absorbed the ideas and enthusiasms of holographers with difficulty. In response, these budding organizations mounted education campaigns that sapped more traditional profit-making activities. These oftrepeated initiatives appear to have had only a local and transient impact. While the early 1970s had witnessed sustained growth in the constituencies of holography, signs of decline in institutional support of display holography became noticeable during the 1990s. ${ }^{64}$

\section{Conclusions: Evaluating Progress, Success and Failure}

As the cognitive boundaries of a technical subject shift, so, too, do its applications and users, and their criteria of success. Examples abound in holography of how 'failures' and 'successes' were interpreted inconsistently by shifting audiences. The various advocates of holography had distinctive aspirations, employed contrasting criteria to evaluate its goals, problems and solutions, and thereby buttressed their own differentiation. Thus Gabor's wavefront reconstruction was typecast as a technically constrained, and even backward-looking, microscopy during the 1950s, unworthy of forecasts. During the 1960s, the revitalized subject was widely understood in terms of photography, an 
analogy that directed predictions in ways that were difficult to sustain. Scientists, artists and artisans portrayed their subject as potential-filled, and judged it by its expansion, especially by the number of adopters. They had divergent definitions of good imagery, however, and so judged progress in conflicting ways. Was the technique developing toward metrological accuracy in a laboratory environment, colorful displays in shop windows, aesthetically nuanced fine art, the recording of public events, or a ubiquitous anti-forgery product? Given the multiple constituencies, no consensus was possible, nor can any generally agreed attribution of progress be made. For the same reason, we cannot judge straightforward technological failure here. There was, however, a failure of technological forecasting, owing to over-confidence in short-term achievements made in an over-inflated funding environment.

Judging progress, success and failure is further complicated by the altering prominence of these marginal technical communities. Embossed holography could be represented as an unalloyed success in the late 1990s not only because of its commercial profitability, but also because there were then fewer holographic artists to criticize its imaging characteristics than two decades earlier. Holography as a concept and technique was discordantly categorized by its users, successively rejected, resurrected and relegated to vulnerable commercial niches.

The history of the assessments and forecasts of holography has implications for other studies in the history of science and technology. As this case shows, historical evaluations of progress can be critically sensitive to appraisals made by different communities, particularly for unstable technologies that are adopted by distinct social groups. Each of them—such as scientists, the military, artists, businesspeople and the publicmay employ different criteria in judging the subject. While we may expect attributions of progress to depend on established or enunciated criteria, the case of holography shows that judgments may be based almost entirely on implicit assumptions and superficial analyses. 65

Expectations for the trajectory of holography were supported by faith in both philosophical positivism and technological progressivism and fueled by the expansive funding environment of 1960s America. The predictions of progress relied on littleexamined assumptions and short-term forecasting, and its monitoring flavored subsequent judgments of success and failure, but reexamination of such assessments is difficult for such insecure subjects: lack of market success or professionalization can hinder the documentation of a field. Would-be fields like holography must be tracked by the historian as they evolve, not from scanty archival records. There will be a tendency to under-represent subjects that have not been judged progressive and successful by its contemporary practitioners and critics.

Holography further illustrates how closure is not an inevitable outcome for debates in scientific subjects that do not reach disciplinary status, or for technologies that do not achieve commercial viability. It suggests caution surrounding uncritical assumptions about the evolution of technological subjects: the inconsistent assessments of progress and success cannot be attributed merely to the youth of a subject or to inchoate relevant social groups. The notion of the 'maturity' of a subject is problematic and must be divorced from scholars' own expectations of progress towards consensus. 
Not all technologies become black-boxed; some merely lose their supporters and are forgotten.

Gabor, the originator of the hologram, wrote and lectured on science and society in his later years. One of his books, Inventing the Future, argued that while technological societies find themselves unable to predict the future, they can invent it for themselves. ${ }^{66}$ This aim, echoed by the engineers at Conductron and the MIT Media Lab, was not reached, but in altered form, the claim can be applied to predictions about the subject that Gabor initiated: the imagined future for holography has been recast repeatedly by successive waves of holographers, and continues to be reinvented by its subsequent practitioner communities and adopters. The complementary perspective-namely explaining the past course of the subject - is equally a matter of reinvention that must be disentangled from implicit assumptions about progress and success. In a subject riven by contrasting assessments and predictions, I have argued that the only indisputable failures surrounding holography concerned the forecasts themselves.

\section{Acknowledgements}

The author acknowledges with gratitude the financial support of the Carnegie Trust for the Universities of Scotland, the British Academy, the Shearwater Foundation and a grant-in-aid from the Friends of the Center for the History of Physics at the American Institute of Physics, and the very helpful comments of anonymous referees.

\section{Notes}

[1] By contrast, perceptive analyses of 'failure' include, for example, Shinn, 'Failure or Success?;' Elzen, 'The Failure of a Successful Artifact;' Kunkle, 'Technology in the Seamless Web;' Gooday, 'Re-writing the "Book of Blots";' McCray, 'What Makes a Failure?'

[2] See, for example, Joerges and Shinn, Instrumentation for contributions on these subjects denoted 'research-technologies'. On the negotiation of professional identity, see Abbott, The System of Professions.

[3] On the social factors in the closure of debates surrounding technological options, see Pinch and Bijker, 'The Social Construction.' My discussion of holography follows excellent studies of the early history of technologies, such as Bijker's study of the bicycle (ibid.), and Susan Douglas's account of radio (Douglas, Inventing American Broadcasting). However, it differs from such cases in dealing with a technology that has not achieved a consensual evaluation by any user group for more than a brief period.

[4] Dennis Gabor to M. E. Haine, letter, 18 June 1949.

[5] British patent 685,286 'Improvements in and Relating to Microscopy', filed 17 December 1947 and published 31 December 1952. Gabor never sought a patent for wavefront reconstruction itself. For his most detailed presentation of the concept, see Gabor, 'Microscopy by Reconstructed Wavefronts.'

[6] These included Gordon L. Rogers, a former student of William Bragg in England; Paul Kirkpatrick and his students Albert V. Baez and Hussein M. A. El-Sum in California; and Adolf W. Lohmann in Germany.

[7] That is, the electron-beam source did not have an adequately narrow range of wavelengths and a localized spatial origin to form many interference fringes. The typical coherent optical 
source of the period was a mercury arc lamp shining through an optical filter and small aperture. This generated interference fringes between two beams for optical path differences of a fraction of a millimeter.

[8] Dennis Gabor to T. E. Allibone, letter, 22 March 1954; Dennis Gabor to Sunday Times, letter, 14 April 1968.

[9] Gordon L. Rogers to A. V. Baez, letter, 19 July 1956.

[10] Allibone, 'White and Black Elephants.'

[11] For a more extensive discussion of Gabor's work and its early reception, see Johnston, 'From White Elephant to Nobel Prize.'

[12] Their principal experimental innovations were the use of 'off-axis' beams, which amounted to imposing a carrier frequency on the optical interference patterns of the hologram, and the use of diffuse, instead of specular, illumination for their subjects.

[13] Leith and Upatnieks, 'New Techniques;' Leith and Upatnieks, 'Reconstructed Wavefronts.'

[14] 'Lensless photography' had been coined for a press release by the American Institute of Physics (AIP) in December 1963 to announce the Leith-Upatnieks grayscale image reconstructions (American Institute of Physics, 'Press release: Lensless optics system makes clear photographs', 5 December 1963; American Institute of Physics, 'Press release: Objects behind others now visible in 3-D pictures made by new method', 25 October 1964).

[15] On the perceived links between holography and photography and their consequences, see Johnston, 'Absorbing New Technologies.'

[16] Denisyuk, 'On Reflection of the Optical Properties;' Denisyuk, 'On Reflection of the Optical Properties-II.'

[17] The optical arrangements for Gabor, Denisyuk and Leith-Upatnieks holograms are not dramatically different. Each technique uses two beams of coherent light, a 'reference' (unperturbed) beam and an 'object' (sample-perturbed) beam, which are recombined on the photographic plate. Gabor's 'in-line' method used a single beam to serve both purposes, with the small object casting its shadow on the plate; Leith's and Upatnieks' 'off-axis' arrangement used part of the light beam to bounce off the sample and onto the plate, and the rest to illuminate the plate directly; and Denisyuk passed the light beam through the photographic plate to reflect off the object, and back onto the plate again. The angular differences between the beams in these three techniques nevertheless yield reconstructions having dramatically different characteristics.

[18] Nye, American Technological Sublime. Nye does not precisely define the 'technological sublime' but offers examples of sublime objects which he says 'cannot be comprehended through words and images alone' (p. xi).

[19] A classic study on optical phenomena in nature is Minnaert, The Nature of Light.

[20] Johnston, 'Telling Tales.'

[21] K. M. Siegel (d. 1975) joined Willow Run as a Research Associate with a BS degree from Rensselaer Polytechnic Institute, and completed an MS there in 1950.

[22] Senior, 'Radiation Lab History.'

[23] Gary D. Cochran, 6 and 8 September 2003, Ann Arbor, MI.

[24] Clark Charnetski, 3 September 2003, Ann Arbor, MI; Cochran and Buzzard, 'The New Art of Holography.'

[25] See Cochran note 23. Reasons for the limited interest in such holograms are discussed below.

[26] Keeve M. Siegel, 'Speech to Conductron Missouri.'

[27] Larry Siebert, 4 September 2003, Ann Arbor, MI. This disparity between the technical requirements and plausibly achievable 'progress' is reminiscent of the later Strategic Defense Initiative ('Star Wars' project) promoted by physicist Edward Teller, which also relied on pulsed lasers.

[28] Siebert, 'Large-Scene Front-Lighted Hologram.' A reflection hologram was made the following year by Denisyuk's team [Staselko et al., 'Holographic Portrait'].

[29] Charnetski, 'The Impact of Holography.'

[30] Goldberg, 'Conductron and McDonnell Douglas.' 
[31] The company's internal reports were uniformly optimistic during the late 1960s. See Wilfong, 'Hologram Product Display;' Wilfong, 'Holography Use Increases.' For the military underpinnings of laser research, see Forman, 'Inventing the Maser' and Seidel, 'From Glow to Flow.'

[32] See, for example, del Sesto, 'Wasn't the Future.'

[33] Charnetski, 'The Impact of Holography.'

[34] The introduction of the stereoscope during the 1850s mirrored aspects of the introduction of holography over a century later. Stereoscopic images were as awe-inspiring and shocking as holograms were, provoking 'at once wonder, exuberance, hesitation and confusion' [Green, " "Pasteboard Masks", quotation p. 109]. In both Europe and America, analysts wrote oftencontradictory explanations of the nature, possibilities and power of the new photographic process, which mutated rapidly from a scientific curiosity to a commercial phenomenon.

[35] Johnston, 'Shifting Perspectives.'

[36] A related constituency was the amateur enthusiast. This was promoted by popular articles in Scientific American, via its 'Amateur Scientist' section, which described scientific construction and experimental projects, and by a handful of articles in journals for physics educators. These appealed to lone experimenters who modelled their activities on a scaled-down version of professional scientific practice and research. As hobbyists rather than goal-directed career workers, amateur holographers tended towards an optimistic and uncritical view of technological progress. See, for example, Heumann and Stong, 'The Amateur Scientist;' Webb, 'Holography for the Sophomore Laboratory;' Dickson and Stong, 'The Amateur Scientist;' Walker, 'Amateur Scientist: Easy Way;' Walker, 'Amateur Scientist: Rainbow;' Walker, 'Amateur Scientist: How to Stop.'

[37] Johnston, 'Reconstructing the History.'

[38] Pinch and Bijker, 'The Social Construction.'

[39] Leith and Upatnieks, trying lasers for the first time in December 1962, found them a nuisance because of the optical 'noise' from stray reflection and interference, and only after several months did they abandon mercury lamps [Hecht, 'Applications Pioneer Interview;' Juris Upatnieks, 12 January 2005].

[40] Speckle is most noticeable from a fixed position and using a small camera aperture to photograph an image having a large depth of field. For observers bobbing their heads to see the parallax of the image, though, the speckle is smeared out.

[41] For a survey, see Bjelkhagen, 'Holographic Portraits.'

[42] Rosen, 'Hologram of the Aerial Image.'

[43] Benton, 'Hologram Reconstructions.'

[44] Brand, The Media Lab.

[45] Yu. N. Denisyuk, 3 May 2003. Rainbow holograms also are devoid of vertical parallax: when moving up and down, the observer sees the same image in a different color rather than a different perspective.

[46] McGrew, 'Mass Produced Holograms.' See, for example, the National Geographic Magazine covers of March 1984, November 1985 and December 1988. Key aspects of embossing technology had been developed for RCA Selectavision Holotape in the early 1970s.

[47] The economics of embossed holograms did not improve the professional situation of holographers: embossing processes were taken over by commercial printing companies using fairly conventional equipment, and relied on holographers only for the production of the original master hologram.

[48] Credit card holograms were introduced by MasterCard in 1983. The hologram industry was subsequently dominated by packaging and security applications, a domain represented by a periodical (Holography News, published by Reconnaissance International from 1987), by annual conferences (Holopack-Holoprint, from 1989) and a trade body (International Hologram Manufacturers' Organization, 1992) seeking to monitor and regulate an industry growing most rapidly in the Far East.

[49] Margaret Benyon, 21 January 2003, Santa Clara, CA. 
[50] Prognostications of a positive future include Weitzman, 'Optical Technologies;' Johnson and Briggs, 'Holography as Applied;' Lang and Eschler, 'Gigabyte Capacities.' For a sociological study of this branch of holographic research, see Tchalakov, 'Innovating in Bulgaria.'

[51] Kramer, 'Holography.'

[52] See, for example, reviews by Gabor, 'The Outlook;' Gabor, 'Holography, Past, Present and Future;' Hammond, 'Holography: Beginnings of a New Art Form;' Dolgoff, 'Commercial Holography;' Denisyuk, 'Holography and Its Prospects;' de Marrais, 'Holography in the Future Tense;' Jeong, 'Future Holography.'

[53] McCluskey, 'What a Mess.'

[54] Dennis Gabor's 1971 Nobel Prize for holography provided a convenient perspective from which to evaluate the subject's past and future. So, too, did the mid-1970s (the end of the 'first decade'), the mid 1980s (the end of the 'second decade') and the 1990s, when the elder statesmen of the subject began to retire. Examples of these waves of historiography include Leith and Upatnieks, 'Holography at the Crossroads;' Gabor, 'Holography, 1948-1971;' Benton, 'Holography: the Second Decade;' Benton, 'Ten Years of White-Light Holography;' Wesly, 'Silver Anniversaries;' Denisyuk, 'My Way in Holography;' and Cross and Cross, 'HoloStories.'

[55] For example, Bringolf, 'Holography: A Medium.'

[56] See also Pizzanelli, 'Evolution of the Mythical Hologram.'

[57] On the reputed public 'misunderstanding' of holograms, and the consequential difficulty of marketing them, see Alexander, 'Seller Beware.'

[58] Milburn, 'Nanotechnology,' quotation p. 263.

[59] A range of cases are discussed in Corn, Imagining Tomorrow and Sturken et al., Technological Visions.

[60] Gary Zellerbach, 1980, Los Angeles; Gary Zellerbach, 2 August 2003.

[61] See, for example, Speer, 'Before Holography.'

[62] A few accredited post-graduate programs appeared, notably at the Royal College of Art (London), Media Lab at MIT (Cambridge, MA), Art Institute of Chicago and Ontario College of Art (Toronto) during the mid 1980s and early 1990s.

[63] MIT Museum, MoH archive series 3, boxes 26-31.

[64] The New York Museum of Holography closed in 1992, and its holdings were auctioned and transferred to the MIT Museum a year later; the Museum für Holographie und neue visuelle Medien in Pulheim, Germany, founded in late 1979, closed in 1994, as did Le Musée de l'Holographie in Paris, founded in 1980; The Holography Unit of the Royal College of Art, an important source of postgraduate fine-art holographers, closed in 1994; the Canada Council ceased funding for holography in 1995; and, the final Gordon Research Conference of scientist-holographers, initiated in 1972, was held in 1997.

[65] Another example is the case of the New National Telescope, which astronomers widely judged a failure because it was never built (McCray, 'What Makes a Failure?). McCray, by contrast, suggests that the project could be deemed a success because of its liberating effect on telescope design, on promotion of international cooperation, and on public promotion of astronomy. Similarly, Elzen argues that the Svedberg ultracentrifuge was seen as a successful artefact by his contemporaries despite its lack of influence on present-day designs (Elzen, 'The Failure of a Successful Artifact').

[66] Gabor, Inventing the Future.

\section{References}

Abbott, Andrew Delano. The System of Professions: An Essay on the Division of Expert Labor. Chicago: University of Chicago Press, 1988.

Alexander, Kent. 'Seller Beware.' Holosphere 13 (1985): 10. 
Allibone, T. E. 'White and Black Elephants at Aldermaston.' Journal of Electronics and Control 4 (1958): 179-192.

Benton, Stephen A. 'Hologram Reconstructions with Extended Incoherent Sources', Paper presented at 1969 annual meeting of the Optical Society of America, 1969.

—. 'Holography: The Second Decade.' Optics News (1977): 16-21.

_ _. 'Ten Years of White-Light Holography', Paper presented at 'Electro-optics/Laser International 1980', London, UK, 1980.

Bjelkhagen, Hans I. 'Holographic Portraits Made by Pulse Lasers.' Leonardo 25 (1992): 443-448.

Brand, Stewart. The Media Lab: Inventing the Future at MIT. New York: Viking, 1987.

Bringolf, Peter H. 'Holography: A Medium in the Making.' Proceedings of the SPIE The International Society for Optical Engineering 2043 (1994): 319-321.

Charnetski, Clark. 'The Impact of Holography on the Consumer', Paper presented at EASCON Convention, Washington DC, 1970.

Cochran, Gary D., and Robert D. Buzzard. 'The New Art of Holography.' In Science Year: The World Book Science Annual 1967. Chicago: Field Enterprises, 1967, 200-11.

Corn, Joseph, ed. Imagining Tomorrow: History, Technology and the American Future. Cambridge MA: MIT Press, 1986.

Cross Lloyd G., and Cecil Cross. 'HoloStories: Reminiscences and a Prognostication on Holography.' Leonardo 25 (1992): 421-4.

de Marrais, Robert. 'Holography in the Future Tense.' Holosphere 12 (1984): 4, 6-7.

del Sesto, Steven L. 'Wasn't the Future of Nuclear Engineering Wonderful?' In Imagining Tomorrow: History, Technology, and the American Future, edited by J. J. Corn. Cambridge MA: MIT Press, 1986, 58-76.

Denisyuk, Yu. N. 'On Reflection of the Optical Properties of an Object in Wavefield of Radiation Scattered by It.' Optika i Spektroskopija 15 (1963): 522-32.

—. 'On Reflection of the Optical Properties of an Object in Wavefield of Radiation Scattered by It-II.' Optika i Spektroskopija 18 (1965): 276-83.

—. 'Holography and its Prospects (Review).' Journal of Applied Spectroscopy 33 (1980): 901-15.

. 'My Way in Holography.' Leonardo 25 (1992): 425-30.

Dickson, Leroy D., and C. L. Stong. 'The Amateur Scientist: Stability of the Apparatus: Insuring a Good Hologram by Controlling Vibration and Exposure.' Scientific American, July 1971: $110-12$.

Dolgoff, E. 'Commercial Holography.' Optical-Spectra 9 (1975): 26-31.

Douglas, Susan J. Inventing American Broadcasting: 1899-1922. Baltimore, MD: Johns Hopkins University Press, 1987.

Elzen, Boelie. 'The Failure of a Successful Artifact: The Svedberg Ultracentrifuge.' In Center on the Periphery: Historical Aspects of 20th-Century Swedish Physics, edited by S. Lindqvist. Canton, MA: Science History Publications, 1993, 347-77.

Forman, Paul. 'Inventing the Maser in Postwar America.' Osiris 7 (1992): 105-34.

Gabor, Dennis. 'Microscopy by Reconstructed Wavefronts.' Proceedings of the Royal Society of London A 197 (1949): 454-87.

- Inventing the Future. London: Secker \& Warburg, 1963.

—. 'The Outlook for Holography.' Optik 28 (1969): 437-41.

—. 'Holography, Past, Present and Future.' Proceedings of the SPIE: The International Society for Optical Engineering 25 (1971): 129-34.

—. 'Holography, 1948-1971.' Science 177 (1972): 299-313.

Goldberg, Shoshanah. 'Conductron and McDonnell Douglas,' Holosphere 15 (1987): 16-18.

Gooday, Graeme. 'Re-writing the 'Book of Blots': Critical Reflections on Histories of Technical Failure.' History and Technology 14 (1998): 265-91.

Green, Harvey. '“Pasteboard masks”: The Stereograph in American Culture 1865-1910.' In Points of View: The Stereograph in America - A Cultural History, edited by E. Earle. New York: Visual Studies Workshop Press, 1979, 109-115. 
Hammond, A. L. 'Holography: Beginnings of a New Art Form or At Least of An Advertising Bonanza.' Science 180 (1973): 484-5.

Hecht, Jeff. 'Applications Pioneer Interview: Emmett Leith.' Lasers \& Applications, 5 April 1986: $56-8$.

Heumann, Sylvain M., and C. L. Stong. 'The Amateur Scientist: How to Make Holograms and Experiment with Them or with Ready-Made Holograms.' Scientific American, February 1967: $122-8$.

Jeong, Tung H. 'Future Holography.' Holosphere 12 (1984): 18.

Joerges, Bernward, and Terry Shinn, ed. Instrumentation: Between Science, State and Industry. Dordrecht: Kluwer, 2001.

Johnson, C., and E. Briggs. 'Holography as Applied to Information Storage and Retrieval Systems.' Journal of the American Society for Information Science 22 (1971): 187-92.

Johnston, Sean F. 'Reconstructing the History of Holography.' Proceedings of the SPIE-The International Society of Optical Engineering 5005 (2003): 455-464.

— . 'Telling Tales: George Stroke and the Historiography of Holography.' History and Technology 20 (2004): 29-51.

—. 'Absorbing New Technologies: Holography as an Analog of Photography.' Physics in Perspective 7 (2005) (in press).

—. 'Shifting Perspectives: Holography and the Emergence of Technical Communities.' Technology \& Culture 46 (2005): 77-103.

—. 'From White Elephant to Nobel Prize: Dennis Gabor's Wavefront Reconstruction.' Historical Studies in the Physical and Biological Sciences (in press).

Kramer, H. 'Holography: A Technical Stunt.' New York Times, 20 July 1975, D 1-2

Kunkle, Gregory C. 'Technology in the Seamless Web: "Success" and "Failure" in the History of the Electron Microscope.' Technology and Culture 36 (1995): 80-103.

Lang, M. and H. Eschler. 'Gigabyte Capacities for Holographic Memories.' Optics and Laser Technology 6 (1974): 219-24.

Leith, Emmett N., and Juris Upatnieks. 'New Techniques in Wavefront Reconstruction.' Journal of the Optical Society of America 51 (1961): 1469.

- 'Reconstructed Wavefronts and Communication Theory.' Journal of the Optical Society of America 52 (1962): 1123-30.

—. 'Holography at the Crossroads.' Optical Spectra 4 (1970): 21.

McCluskey, Caroline. 'What a Mess.' Wavefront 2 (1987): 4.

McCray, Patrick. 'What Makes a Failure? Designing a New National Telescope, 1975-1984.' Technology and Culture 42 (2001): 265-91.

McGrew, Steve. 'Mass Produced Holograms for the Entertainment Industry.' Proceedings of the SPIE 391 (1983): 19-20.

Milburn, Colin. 'Nanotechnology in an Age of Posthuman Engineering: Science Fiction as Science.' Configurations 10 (2002): 261-95.

Minnaert, M. The Nature of Light and Colour in the Open Air. New York: Dover, 1954.

Nye, David E. American Technological Sublime. Cambridge, MA: MIT Press, 1994.

Pinch, Trevor J., and Wiebe E. Bijker. 'The Social Construction of Facts and Artifacts: Or How the Sociology of Science and the Sociology of Technology Might Benefit Each Other.' In The Social Construction of Technological Systems. edited by W. E. Bijker, T. P. Hughes and T. J. Pinch. Cambridge, MA: MIT Press, 1987, 17-50.

Pizzanelli, David. 'Evolution of the Mythical Hologram.' Proceedings of the SPIE The International Society for Optical Engineering 1732 (1992): 430-7.

Rosen, L. 'Hologram of the Aerial Image of a Lens.' Proceedings of the IEEE 54 (1966): 79-80.

Seidel, Robert W. 'From Glow to Flow: A History of Military Laser Research and Development.' Studies in the History of the Physical and Biological Sciences 17 (1986): 111-148.

Senior, T. B. A. 'Radiation Lab History', http://www.eecs.umich.edu/RADLAB/labhistory.html, last updated 2003; accessed 1 September 2004. 
Shinn, Terry. 'Failure or Success? Interpretations of 20th Century French Physics.' Historical Studies in the Physical and Biological Sciences 16 (1986): 353-69.

Siebert, L. D. 'Large-Scene Front-Lighted Hologram of a Human Subject.' Proceedings of the IEEE 56 (1968): 1242-3.

Speer, L. 'Before Holography—A Call for Visual Literacy.' Leonardo 22 (1989): 299-306.

Staselko, D. I., Yu. N. Denisyuk and A. G. Smirnov. 'Holographic Portrait of a Man.' Zhurnal Nauch i Prikl Photogr i Kinematogra 15 (1970): 147.

Sturken, Marita, Douglas Thomas and Ball-Rokeach, ed. Technological Visions: The Hopes and Fears That Shape New Technology. Philadelphia, PA: Temple University Press, 2004.

Tchalakov, Ivan. 'Innovating in Bulgaria-Two Cases in the Life of a Laboratory Before and After 1989.' Research Policy 30 (2001): 391-402.

Walker, Jearl. 'Amateur Scientist: Easy Way to Make Holograms.' Scientific American, February 1980: 158.

—. 'Amateur Scientist: How to Stop Worrying About Vibrations and Make Holograms Viewable in White Light.' Scientific American, May 1989: 134.

—_. 'Amateur Scientist: Rainbow Holograms.' Scientific American, Sep 1986: 114.

Webb, Robert H. 'Holography for the Sophomore Laboratory.' American Journal of Physics 36 (1968): 62-3.

Weitzman, C. 'Optical Technologies for Future Computer System Design.' Computer Design 9 (1970): 169-75.

Wesly, Ed. 'Silver Anniversaries.' Holosphere 15 (1988): 12-13.

Wilfong, Joan. 'Hologram Product Display Announced.' Conductron Antenna, 2 December 1968: 4.

—_. 'Holography Use Increases.' Conductron Antenna, 3 December 1969: 4.

\section{Archives}

Archival sources cited here include Imperial College (IC) and the Science Museum (Sci Mus), both in London, the Museum of Holography archive of the Massachusetts Institute of Technology Museum (MIT), Cambridge, MA, the Ross Collection (Studio 286, London) and the SFJ Collection (University of Glasgow, Crichton Campus, Dumfries).

Benyon, Margaret. 21 January 2003, Santa Clara, CA, interview communication to SFJ, SFJ collection. Charnetski, Clark. 3 September 2003, Ann Arbor, MI, interview communication to SFJ, SFJ collection. Cochran, Gary D. 6 and 8 September 2003, Ann Arbor, MI, interview communication to SFJ, SFJ collection.

Denisyuk, Yu. N. 3 May 2003, email communication to SFJ, SFJ collection.

Gabor, Dennis to M. E. Haine, letter, 18 June 1949. Rugby. IC GABOR MA/6/1.

Gabor, Dennis to T. E. Allibone, letter, 22 March 1954. IC GABOR MA/6/1.

Gabor, Dennis to Sunday Times, letter, 14 April 1968. IC B/GABOR/11/111.

Rogers, Gordon L. to A. V. Baez, letter, 19 July 1956. Sci Mus ROGRS 6.

Siebert, Larry. 4 September 2003, Ann Arbor, MI, interview communication to SFJ, SFJ collection.

Siegel, Keeve M. 'Speech to Conductron Missouri.' Audio recording, 1966, SFJ collection.

Upatnieks, Juris. 12 January 2005, email communication to SFJ, SFJ collection.

Zellerbach, Gary. 1980, Los Angeles, interview communication to Jonathan Ross, Ross collection.

Zellerbach, Gary. 2 August 2003, email communication to SFJ, SFJ collection. 